\title{
Geopolímeros basados en residuos de la producción de áridos ligeros
}

\section{Geopolymerization of lightweight aggregate waste}

\author{
P. Soares ${ }^{(*)}$, A. T. Pinto(**), V. M. Ferreira(*), y J. A. Labrincha ${ }^{(*)}$
}

Recepción/Received: 22-I-07

Aceptación/Accepted: 1-VIII-07

Publicado online/Online publishing: 30-V-08

\section{RESUMEN}

La geopolimerización es una manera viable para procesar y agregar valor a los residuos industriales de alumino-silicato dando lugar a materiales con elevadas resistencias mecánmicas, alta inercia química y que permiten encapsular otros residuos, incluso peligrosos.

Los residuos industriales que proceden de diversos tipos de arcillas para la fabricación de áridos ligeros se han caracterizado para la producción de geopolímeros mediante el proceso de ataque alcalino. Su incorporación en una matriz geopolimérica permite la posibilidad de desarrollo de paneles de aislamiento (térmico y acústico). Además, la inmovilización de materiales peligrosos es un logro adicional importante.

Los geopolímeros se han producido con fórmulas diferentes y se han caracterizado sus propiedades mecánicas, composición y microestructura, para dar lugar a una composición interesante con propiedades aislantes. Se ha llevado a cabo además un análisis preliminar sobre la formación de sales que tiene lugar en este tipo de geopolímeros como una desventaja de los mismos.

Palabras clave: geopolímeros, activación alcalina, áridos ligeros, reciclado.

\section{SUMMARY}

Geopolymerization is a viable way to process and re-use alumino-silicate industrial waste while producing highstrength, high chemical inertia materials that can effectively immobilize other industrial by-products, and even hazardous waste.

In this study industrial waste from different stages of the manufacture of lightweight expanded clay aggregate was characterized for its possible transformation, via alkali activation, to geopolymers. The ultimate aim was to assess the possibility of using such geopolymers to develop thermal and acoustic insulation panels. The containment of hazardous materials is another important application for these new materials.

Geopolymers were prepared for this study with different particles size distributions and activator concentrations. Their mechanical properties, composition and microstructure were characterized and a material with promising insulating properties was produced. A preliminary analysis was conducted of the salt formation observed in these geopolymers, the chief drawback to their use.

Keywords: geopolymers, alkali activation, lightweight aggregates, recycling.

(*) CICECO, Universidad de Aveiro,(Aveiro, Portugal).

(**) Universidad de Trás-os-Montes e de Contralto Douro, (Vila Verdadero, Portugal). 


\section{INTRODUCCIÓN}

Desde hace unos años crece el interés sobre la necesidad de que el tratamiento, la eliminación o la valorización de residuos como una consecuencia del incremento de la conciencia hacia la seguridad del medioambiente. El aumento del consumo y el aumento consiguiente de la producción industrial ha llevado a una disminución rápida de los recursos naturales disponibles y, al mismo tiempo, a elevado volumen de producción de residuos o subproductos que se generan (1). Las investigaciones recientes han dado lugar a la propuesta de la valorización de residuos incluyendo, por ejemplo, la posibilidad de utilizarlos en la fabricación de materiales $(2,3)$. La arcilla expandida ligera tiene una tasa de producción muy elevada. Un horno típico para este tipo de áridos suele producir alrededor de 50 tons/ hora (1), generando una cantidad grande de residuos que tendrían una potencial valorización. Una mezcla de arcilla plástica, agua y aditivos aseguran la expansión durante el proceso en un horno giratorio. El procedimiento de mezcla previo es parecido al de la fabricación de ladrillos como materiales cerámicos. Los áridos ligeros, que tienen muchas aplicaciones para la fabricación de materiales de construcción, se obtienen y aplican según el tamaño de partícula. No obstante, queda todavía una cantidad considerable de áridos ligeros generados, con un tamaño menos de $2-3 \mathrm{~mm}$, que se observa como residuos desde el punto de vista tradicional de sus aplicaciones.

La geopolimerización surge como un proceso viable para transformar estos residuos residuos en nuevos productos y valorizarlos como materias primas. Los geopolímeros tienen la fórmula empírica general: $\mathrm{M}_{\mathrm{n}}\left(-\left(\mathrm{Si}-\mathrm{O}_{2}\right)_{\mathrm{z}}-\mathrm{Al}-\right.$ $\mathrm{O})_{n} \cdot \mathrm{wH}_{2} \mathrm{O}$, donde $\mathrm{M}$ es un catión alcalino $(\mathrm{K}, \mathrm{Na}$, $\mathrm{Li}$ o $\mathrm{Cs})$, $z$ vale comúnmente 1,2 ó 3 y $n$ es el grado de polimerización. Los geopolímeros se forman por la co - polimerización del aluminio y especies de silicato que proceden de la disolución de la alúmina y el silicio presentes en la constitución química de los materiales de origen, a valores altos de $\mathrm{pH}$, en presencia de silicatos solubles alcalinos (4). La geopolimerización es por supuesto dependiente de la composición mineralógica, de la relación sílice/alúmina, de la concentración de álcalis, de la morfología del reactivo y de la superficie específica $(4,5)$. Los áridos ligeros con base en la arcilla contiene ya una proporción rica inicial de $\mathrm{SiO}_{2}$ y $\mathrm{Al}_{2} \mathrm{O}_{3}$.

La formación de zeolitas y de precursores zeolíticos es similar de manera que los Al y Si disponibles en la solución reaccione en medios alcalinos para formar complejos de polihidroxialuminio. Para la formación de zeolitas, el proceso geopolímero requiere de un precursor reactivo y que el proceso esté afectado mayormente por la concentración de reactivo (específicamente $\mathrm{OH}$-) y por el tiempo de curado y las condiciones de temperatura y hume-

\section{INTRODUCTION}

Today's general awareness about environmental issues has led to a growing concern over waste generation and the realization that such materials must be treated, eliminated or re-used. Rising consumption and the concomitant increase in industrial production has occasioned both a rapid decline in natural resources and the generation of large volumes of waste or by-products (1). Recent research into the economically viable re-use of waste has given rise to different proposals, including the possibility of its inclusion in building materials $(2,3)$. The production rate of lightweight expanded clay aggregate is very high. Attending to the 50-ton/hour output of a typical furnace for this type of aggregate (1) there is the generation of vast amounts of potentially re-usable waste. The process involves the expansion of a mix of plastic clay, water and additives during firing in a rotational kiln. Mixing prior to firing is similar to the procedure followed to manufacture other clay-based materials such as brick. Lightweight aggregates, which have many applications in building processes and materials, are graded and commercialized by particle size. Nonetheless, a substantial amount of the lightweight aggregate produced is under 2-3 mm, i.e., too small for traditional applications.

Geopolymerization is a viable process for transforming this waste into new, re-usable raw materials. The general formula for geopolymers is $\mathrm{M}_{n}\left(-\left(\mathrm{Si}-\mathrm{O}_{2}\right)_{z}-\mathrm{Al}-\mathrm{O}\right)_{n} \cdot \mathrm{wH}_{2} \mathrm{O}$, where $M$ is an alkaline cation ( $K, \mathrm{Na}, L I$ or $\mathrm{CS}), z$ is generally assigned a value of 1,2 or 3 and $n$ is the degree of polymerization. Geopolymers are formed via co-polymerization of the aluminium and silicate species resulting from the dissolution of the alumina and silicon present in the original materials, at high $\mathrm{pH}$ values and in the presence of soluble alkaline silicates (4). Geopolymerization depends, of course, on the mineral composition, silica/alumina ratio, alkali concentration, reagent morphology and BET specific surface of the materials involved $(4,5)$. Clay-based lightweight aggregate has a high initial content of $\mathrm{SiO}_{2}$ and $\mathrm{Al}_{2} \mathrm{O}_{3}$.

In zeolite and zeolite precursor formation, the $\mathrm{Al}$ and $\mathrm{Si}$ available in the solution react in an alkaline medium to form polyhydroxyaluminosilicate complexes. Similarly, geopolymerization calls for a reactive precursor and is affected primarily by reagent (especially $\mathrm{OH}$-) concentration and curing conditions: time, temperature and relati- 
dad relativa. Sin embargo, existen claras diferencias en estos dos de tipos de productos, el uno principales es el carácter cristalino de la estructura de las zeolitas estructura mientras que los geopolímeros son generalmente amorfos $(6,7)$.

Los materiales zeolíticos son conocidos por su capacidad de cambio iónico que permite a los residuos químicos tóxicos el ser absorbidos. Los geopolímeros se comportan de forma similar las zeolitas, inmovilizando elementos peligrosos dentro de su matriz, y también convirtiendo a los residuos semisólidos en residuos sólidos inertizados (6). Por lo tanto, los elementos peligrosos presentes en los residuos puede ser encapsulados en la red tridimensional de una matriz geopolimérica (8-10). Otra ventaja es su relativa alta estabilidad y resistencia a la erosión detectada en morteros y cementos antiguos y también en los modernos cementos de geopolímeros (11).

En este trabajo, los residuos de la producción de áridos ligeros se transforman en geopolímeros mediante la activación alcalina y luego se caracterizan desde el punto de vista de sus propiedades mecánicas, composición y microestructura. El objetivo es construir materiales en la forma de paneles para mejorar el aislamiento térmico y acústico. Otras aplicaciones que hagan uso de su elevada resistencia química puede constituir también un enfoque alternativo.

\section{EXPERIMENTAL}

Los residuos estudiados en el este trabajo proceden de diversas partes del ciclo de producción de áridos ligeros. En su mayor parte están constituidos por partículas finas (polvo de aspiración de filtros y polvos de tamizado) o de rechazo de los áridos (gránulos no - conformados) o incluso fracción graduada $<3 \mathrm{~mm}$ con una aplicación reducida en el mercado actual.

Tomando en cuenta la generación y cadencia de producción de fracciones diferentes, algunas composiciones se han podido formular combinando diferentes residuos de áridos ligeros. Así, la mezcla 1 involucra a residuos en la misma cantidad que se generan en la producción rutinaria (89\% la polvos de aspiración $+7 \%$ de gránulos $+4 \%$ polvos de tamizado). la Mezcla 2 contiene $50 \%$ de polvo fino ( $90 \%$ de polvos de aspiración y $10 \%$ de polvos de tamizado) y 50\% de áridos de la fracción de 0-3 mm. Los componentes se mezclaron en seco durante una hora. Los materiales en bruto (residuos de áridos ligeros) se caracterizaron desde el punto de vista de distribución del tamaño de partícula, composición química y mineralógica. La composición mineralógica se obtuvo por difracción de Rayos X (XRD, Rigaku Geigerflex D/max - Serie) y la ve humidity. Clear differences can be identified between these two types of products, however, the chief distinction being that zeolites have a crystalline structure whereas geopolymers are generally amorphous $(6,7)$.

Zeolite materials are known for their ability to exchange ions and therefore to absorb toxic chemical waste. Geopolymers behave in much the same way as zeolites, locking toxic elements in their matrix and converting semi-solid waste into "adhesive" solids (6). Consequently, toxic elements present in waste can be contained in the three-dimensional networks that comprise geopolymer matrices (8-10). Another advantage is the proven durability and erosion resistance of both ancient mortars and cements and modern geopolymer cements (11).

In this study, lightweight aggregate waste was alkali activated to form geopolymers, which were in turn analyzed to determine their strength, composition and microstructure. The aim was to lay the ground for the development of thermal and acoustic insulation panels for use in construction. Other applications capitalizing on the chemical potential of these materials may constitute alternative approaches to their re-use.

\section{EXPERIMENTAL}

The present study used waste from different stages of the lightweight aggregate ( $L W A$ ) production cycle. It consisted largely in fine particles (powder retained in filters and sieves), but also included rejected aggregate (nonconforming particles) and graded fractions $(\varnothing<3 \mathrm{~mm}$ ) with scant market demand at present.

Aggregate blends were prepared for this study bearing in mind output rates and the pace of generation of the different types of LWA waste. Mix 1 contained waste in the proportion generated in standard production, i.e., $89 \%$ powder retained in filters $+7 \%$ rejected particles $+4 \%$ powder retained in sieves. Mix 2 comprised $50 \%$ powder ( $90 \%$ from filters $+10 \%$ from sieves) and $50 \%$ 0/3-mm aggregate. The components were blended dry in a mixer for about one hour. The alkaline activator used to geopolymerize these mixes was $5-M$ or $10-M$ sodium hydroxide (Table 1). Pastes C1, C3 and C4 were made from the activated $L W A$ waste, while paste $C 2$, the reference, was 
composición química por fluorescencia de Rayos X (XRF, Philips X'UNIQUE II). Se realizaron usando hidroxido de sodio al 5 M ó 10 M (Tabla 1) como el activador alcalino. En las composiciones C1, C3 y C4 la matriz se diseña con el máximo contenido de árido ligero activado, mientras que en la C2 se diseña con cemento Portland preparándose simplemente (relación agua/cemento $=0,9$ ) para propósitos de comparación. prepared with a (type I) Portland cement with a w/c ratio of 0.9 .

Tabla 1 / Table 1

Composiciones de los geopolímeros estudiados

Composition of the geopolymers studied Mix $1=89 \%$ filter powder $+7 \%$ rejected particles $+4 \%$ sieve powder. Mix $2=50 \%$ powder (90\% from filters $+10 \%$ from sieves) and $50 \% 0 / 3-\mathrm{mm}$ aggregate.

\begin{tabular}{|c|c|c|c|}
\hline Composición / Paste & Mezcla / Mix & Razón L/S* / L/S ratio* & Activador / Activator \\
\hline C1 & 2 & 0.4 & {$[\mathrm{NaOH}]=10 \mathrm{M}$} \\
\hline C2 & 2 & 0.4 & Water $=0.9 * *$ \\
\hline C3 & 1 & 0.4 & {$[\mathrm{NaOH}]=10 \mathrm{M}$} \\
\hline C4 & 2 & 0.4 & {$[\mathrm{NaOH}]=5 \mathrm{M}$} \\
\hline
\end{tabular}

* Razón de activador (líquido) a matriz (sólido) / Activator (liquid) to matrix (solid) ratio

** Razón Agua/Cemento / Water/cement ratio

Con respecto al proceso alcalino de activación, el proceso de mezcla se hizo con cuidado, introduciendo primero la fase líquida (activador) y luego, lentamente, los residuos sólidos en polvo sólidos, mezclando bien durante 10 minutos hasta que se obtiene un material homogéneo. Para la preparación de las muestras se usaron moldes de $4 \times 4 \times 16 \mathrm{~cm}$ de dimensiones. Después de 24 horas, se desmoldearon las muestras, se protegieron con una película de polietileno y se continuó la cura en una sala acondicionada ( $\mathrm{T}$ aproximada de $25^{\circ} \mathrm{C}$ y humedad relativa del $\sim 50 \%$ ) hasta el momento en que se realiza la caracterización. Los geopolímeros se caracterizaron mecánicamente sobre muestras con diferentes tiempos de curado $(1,7,14$ y 28 días) mediante ensayos a flexión y compresión. Otras técnicas complementarias se usaron para la caracterización de los productos geopoliméricos, específicamente por microscopía electrónica de barrido (SEM, Hitachi S-4100) para la evaluación de la microestructura y DRX para el análisis de las fases. En el caso de la composición (C4), se ha determinado la densidad (por el método de inmersión de líquidos) y la conductividad térmica mediante medidas del coeficiente de conductividad (según ASTM C-518-91 y EN 1530-93) en un equipo Holometrix TCA-200. También se ha realizado el análisis preliminar por DRX de sales formadas en estos geopolímeros.

\section{RESULTADOS Y DISCUSIÓN}

\subsection{Caracterización de las materias primas}

La Tabla 2 presenta la composición mineral del residuo de árido ligero por DRX y en donde es posible observar alfa-
Alkali activation was performed with care, slowly adding the powdery waste to the liquid activator and mixing vigorously until a homogeneous material was obtained. The pastes were then poured into $4 \times 4 \times 16-\mathrm{cm}$ moulds. The specimens were removed from the moulds 24 hours later, wrapped in polyethylene film and cured at ambient temperature and relative humidity $\left(T \sim 25^{\circ} \mathrm{C} ; \mathrm{RH} \sim 50 \%\right)$ until the characterization trials were conducted. The particle size distribution and mineralogical and chemical composition were found for the prime materials (LWA waste). Mineralogy was determined via $X$-ray diffraction $(X R D)$ on a Rigaku Geigerflex D/max-Series diffractometer, and chemical composition by $X$-ray fluorescence (XRF) with a Philips X'UNIQUE II spectrometer. One-, 7-, 14- and 28day geopolymer specimens were tested for bending and compressive strength.. Other characterization techniques were likewise used, including scanning electron microscopy (SEM) with a Hitachi S-4100 microscope to determine microstructure, XRD for phase composition, and in the case of paste C4, liquid immersion to establish density. Conductivity coefficient measurements were also taken (according to ASTM C-518-91 and EN 1530-93 specifications) with a Holometrix TCA-200 heat flow meter to explore insulating properties. Lastly, XRD was also used for a preliminary analysis of the salt forming on these geopolymers.

\section{RESULTS AND DISCUSSION}

\subsection{Characterization of source materials}

Table 2 presents the mineral composition of the various LWA waste materials identified by XRD, where the major 
cuarzo como la fase cristalina más importante. Las otras fases son también las típicas involucradas en la producción de áridos basados en arcillas. crystalline phase was a-quartz. The other phases identified were also typical of clay-based aggregates.

Tabla 2 / Table 2

Análisis mineralógico (DRX) de los residuos de áridos ligeros Mineralogical analysis (XRD) of LWA waste

\begin{tabular}{|c|c|c|c|c|}
\hline $\begin{array}{c}\text { Composición mineralógica } \\
\text { Mineralogical composition }\end{array}$ & $\begin{array}{c}\text { Rechazo } \\
\text { Rejected }\end{array}$ & $\begin{array}{c}\text { Polvo tamizado } \\
\text { Powder retained } \\
\text { on sieves }\end{array}$ & $\begin{array}{c}\text { Polvo aspirado } \\
\text { Powder retained in } \\
\text { filters }\end{array}$ & $\begin{array}{c}\text { Fracción 0/3 mm } \\
\text { 0/3-mm fraction }\end{array}$ \\
\hline$\alpha$ - alfa- cuarzo / $\alpha$-quartz & $* * * *$ & $* * * *$ & $* * * *$ & $* * *$ \\
\hline Illita / Illite & $*$ & & $*$ & $*$ \\
\hline Caolinita / Kaolinite & & & & $*$ \\
\hline Feldespato- K / K-Feldspar & $*$ & $*$ & $* *$ & \\
\hline Calcita / Calcite & & & $* *$ & \\
\hline
\end{tabular}

(**** Intensidad Alta y/o fase cristalina; * Trazas) (**** High Intensity and/or crystalline phase; * Traces)

El análisis del tamaño de partícula se da en la Tabla 3. D50 significa el diámetro mayor del $50 \%$ de las partículas. Los polvos de aspiración, como era de esperar, tienen una distribución más fina de partículas, mostrando unas $13 \mu \mathrm{m}$ de diámetro promedio, considerando la 0/3 fracción (D50 alrededor $450 \mu \mathrm{m}$ ). No obstante, el diámetro promedio (D50) para la partícula más fina LWA de residuos, oscila bastante (entre 13 y $27 \mu \mathrm{m})$.
Table 3 shows the particle size distribution for the LWA waste. D50 symbolizes the median diameter. As might be expected, the filter powder contained the finest particles, with a median diameter of around $13 \mu \mathrm{m}$, while the coarsest distribution was found for the $0 / 3$ fraction (D50 of around $450 \mathrm{~mm}$ ). Despite such a wide range, the finer particle LWA waste materials exhibited fairly similar median diameter values (ranging from 13 to $27 \mu \mathrm{m}$ ).

Tabla 3 / Table 3

Distribución del tamaño de partícula

LWA waste particle size distribution

\begin{tabular}{|c|c|c|c|c|}
\hline \multirow[t]{2}{*}{ Residuo / Waste } & \multicolumn{3}{|c|}{$\begin{array}{l}\text { Distribución de tamaño (\%) } \\
\text { Size distribution (\%) }\end{array}$} & \multirow{2}{*}{$\begin{array}{l}D_{50} \\
(\mu \mathrm{m})\end{array}$} \\
\hline & $(>63 \mu \mathrm{m})$ & $(2-63 \mu \mathrm{m})$ & $(<2 \mu \mathrm{m})$ & \\
\hline Gránulos rechazados / Rejected particles & 44.6 & 45.2 & 10.2 & 26.8 \\
\hline Polvo tamizado / Sieve powder & 26.9 & 70.6 & 2.4 & 22.0 \\
\hline Polvo de aspiración / Filter powder & 11.4 & 85.3 & 3.4 & 13.2 \\
\hline Fracción 0/3 / 0/3-mm fraction & 99.3 & 0.66 & 0.04 & 450 \\
\hline
\end{tabular}

El análisis químico cuantitativo se ha realizado por espectroscopia de FRX para cada conjunto de residuos de áridos ligeros, así como también en las mezclas preparadas. Por lo tanto, se presenta también (Tabla 4) la composición química de las mezclas de estas materias primas, en las proporciones citadas en la sección experimental del proceso. En todos ellos es posible observar un contenido alto de $\mathrm{SiO}_{2}$ y $\mathrm{Al}_{2} \mathrm{O}_{3}$, que evidencia la potencialidad alta de estos materiales para el proceso alcalino de activación, considerando la historia térmica previa del material.
The quantitative chemical composition of each type of LWA waste and the mixes prepared for this study was determined by XRF spectroscopy, as shown in Table 4. With high $\mathrm{SiO}_{2}$ and $\mathrm{Al}_{2} \mathrm{O}_{3}$ contents and given their thermal history, all these materials present a high alkali activation potential. 
El contenido relativamente alto de óxido de calcio se encontró especialmente en los polvos de aspiración en la fracción 0/3 fracción, y, por tanto, en las mezclas preparadas merece también una atención especial. Según algunos autores, la posibilidad de formación de la fase CS-H a partir del óxido de calcio depende de la concentración de $\mathrm{NaOH}$ (el activador) que debe ser moderada (11).
Furthermore, the filter powder as well as the 0/3-mm fraction and, therefore, the mixes, exhibited a relatively high calcium oxide content. Some authors (11) maintain that $\mathrm{C}-\mathrm{S}-\mathrm{H}$ formation from calcium oxide depends on the use of moderate activator $(\mathrm{NaOH})$ concentrations.

Tabla 4 / Table 4

Análisis químico cuantitativo (FRX) del residuo de árido ligero XRF chemical analysis of LWA waste

\begin{tabular}{|c|c|c|c|c|c|c|}
\hline $\begin{array}{c}\text { Oxido (\%) } \\
\text { Oxide (\%) }\end{array}$ & $\begin{array}{c}\text { Gránulos } \\
\text { rechazados } \\
\text { Rejected } \\
\text { particles }\end{array}$ & $\begin{array}{c}\text { Polvo tamizado } \\
\text { Sieve powder }\end{array}$ & $\begin{array}{c}\text { Polvo de aspiración } \\
\text { Filter powder }\end{array}$ & $\begin{array}{c}\text { Fracción 0/3 } \\
\text { 0/3 fraction }\end{array}$ & $\begin{array}{c}\text { Mezcla 1 } \\
\text { Mix 1 }\end{array}$ & $\begin{array}{c}\text { Mezcla 2 } \\
\text { Mix 2 }\end{array}$ \\
\hline $\begin{array}{c}\text { Pérdida por } \\
\text { calcinación / Loss } \\
\text { on ignition }\end{array}$ & 2.2 & 1.1 & 12.0 & --- & -- \\
\hline $\mathrm{SiO}_{2}$ & 57.0 & 56.0 & 39.0 & 45.6 & 38.6 & 46.7 \\
\hline $\mathrm{Al}_{2} \mathrm{O}_{3}$ & 20.0 & 20.0 & 18.0 & 17.1 & 16.8 & 17.2 \\
\hline $\mathrm{Fe}_{2} \mathrm{O}_{3}$ & 7.5 & 7.8 & 5.4 & 11.3 & 8.6 & 11.0 \\
\hline $\mathrm{CaO}$ & 3.7 & 5.3 & 17.0 & 15.2 & 20.7 & 11.1 \\
\hline $\mathrm{MgO}$ & 4.1 & 4.1 & 3.2 & 3.5 & 2.9 & 3.2 \\
\hline $\mathrm{Na}_{2} \mathrm{O}$ & $<0.5$ & $<0.5$ & $<0.5$ & 0.2 & 0.2 & 0.2 \\
\hline $\mathrm{K}_{2} \mathrm{O}$ & 4.1 & 4.1 & 2.4 & 4.7 & 3.5 & 5.1 \\
\hline $\mathrm{TiO}_{2}$ & 0.89 & 0.9 & 0.88 & 1.3 & 1.4 & 1.5 \\
\hline $\mathrm{MnO}_{\mathrm{PnO}}$ & $<0.3$ & $<0.3$ & $<0.3$ & 0.1 & 0.1 & 0.1 \\
\hline $\mathrm{P}_{2} \mathrm{O}_{5}$ & $<0.5$ & $<0.5$ & 0.5 & 0.1 & 0.3 & 0.3 \\
\hline
\end{tabular}

Desde el punto de vista de la composición, la relación más conveniente entre óxidos para el procesamiento del geopolímero debe estar alrededor del 3,3-4,5 $\left(\mathrm{SiO}_{2} / \mathrm{Al}_{2} \mathrm{O}_{3}\right), \quad 0,20-0,48 \quad\left(\mathrm{Na}_{2} \mathrm{O} / \mathrm{SiO}_{2}\right)$ y $0,80-1,60$ $\left(\mathrm{Na}_{2} \mathrm{O} / \mathrm{Al}_{2} \mathrm{O}_{3}\right)$ (12). La Tabla 5 muestra la relación calculada de óxido para las mezclas 1 y 2 y confirma que la relación más importante $\left(\mathrm{SiO}_{2} / \mathrm{Al}_{2} \mathrm{O}_{3}\right)$ se adapta bien dentro de los valores recomendados. Aunque las relaciones para el $\mathrm{Na}_{2} \mathrm{O}$ no son las más adecuadas, estos valores siempre pueden ser corregidos con adiciones de carbonato de sodio si llega a ser realmente necesario para el proceso de formación del geopolímero.
The most suitable oxide ratios for processing geopolymers is thought to be around 3.3-4.5 $\left(\mathrm{SiO}_{2} / \mathrm{Al}_{2} \mathrm{O}_{3}\right), 0.20$ $0.48\left(\mathrm{Na}_{2} \mathrm{O} / \mathrm{SiO}_{2}\right)$ and $0.80-1.60\left(\mathrm{Na}_{2} \mathrm{O} / \mathrm{Al}_{2} \mathrm{O}_{3}\right)$ (12). According to the oxide ratios calculated for mixes 1 and 2 (Table 5), the main ratio $\left(\mathrm{SiO}_{2} / \mathrm{Al}_{2} \mathrm{O}_{3}\right)$ was well within the recommended values in these blends. While the ratios involving $\mathrm{Na}_{2} \mathrm{O}$ were not necessarily optimal for geopolymer formation, they can be readily corrected by adding sodium carbonate as necessary.

Tabla 5 / Table 5

Las relaciones de óxidos de las mezcla de residuos de áridos ligeros

Theoretical oxide ratios in LWA waste mixes

\begin{tabular}{|c|c|c|}
\hline Oxide ratio & Mezcla 1 / Mix 1 & Mezcla 2 / Mix 2 \\
\hline $\mathrm{SiO}_{2} / \mathrm{Al}_{2} \mathrm{O}_{3}$ & 3.89 & 4.60 \\
\hline $\mathrm{Na}_{2} \mathrm{O} / \mathrm{SiO}_{2}$ & 0.005 & 0.003 \\
\hline $\mathrm{Na}_{2} \mathrm{O} / \mathrm{Al}_{2} \mathrm{O}_{3}$ & 0.018 & 0.016 \\
\hline
\end{tabular}


La relación $\mathrm{SiO}_{2} / \mathrm{Al}_{2} \mathrm{O}_{3}$ antes mencionada es una relación nominal y no debería ser confundida con la relación verdadera en los productos de reacción, porque una parte importante del contenido de sílice está en forma del alfacuarzo (que es una fase de baja reactividad). Además, algunos trabajos previos $(5,13)$ han demostrado que en el proceso de disolución de las materias primas, el aluminio se lixivia antes que el silicio, aunque que un proceso sincronizado de rotura tiene lugar y la velocidad de disolución de la sílice tiende a disminuir cuando la fuente de aluminio es consumida totalmente.

\subsection{Caracterización de los productos de reacción}

Después de la activación alcalina de las composiciones C1 a C4 según las condiciones resumidas en la Tabla1, los geopolímeros resultantes han sido caracterizados.
The above-mentioned $\mathrm{SiO}_{2} / \mathrm{Al}_{2} \mathrm{O}_{3}$ ratio is nominal and should not be mistaken for the real ratio in the reaction products, because a significant amount of the silica is found in the form of low reactivity quartz. Moreover, prior studies $(5,13)$ showed that the aluminium leached before the silicon during the dissolution of the source materials, although some synchronization was observed, with the rate of silica dissolution declining after the source of aluminium was depleted.

\subsection{Characterization of the reaction products}

After mixes 1 and 2 were alkali activated and the OPC hydrated, pastes C1 to C4 were characterized.

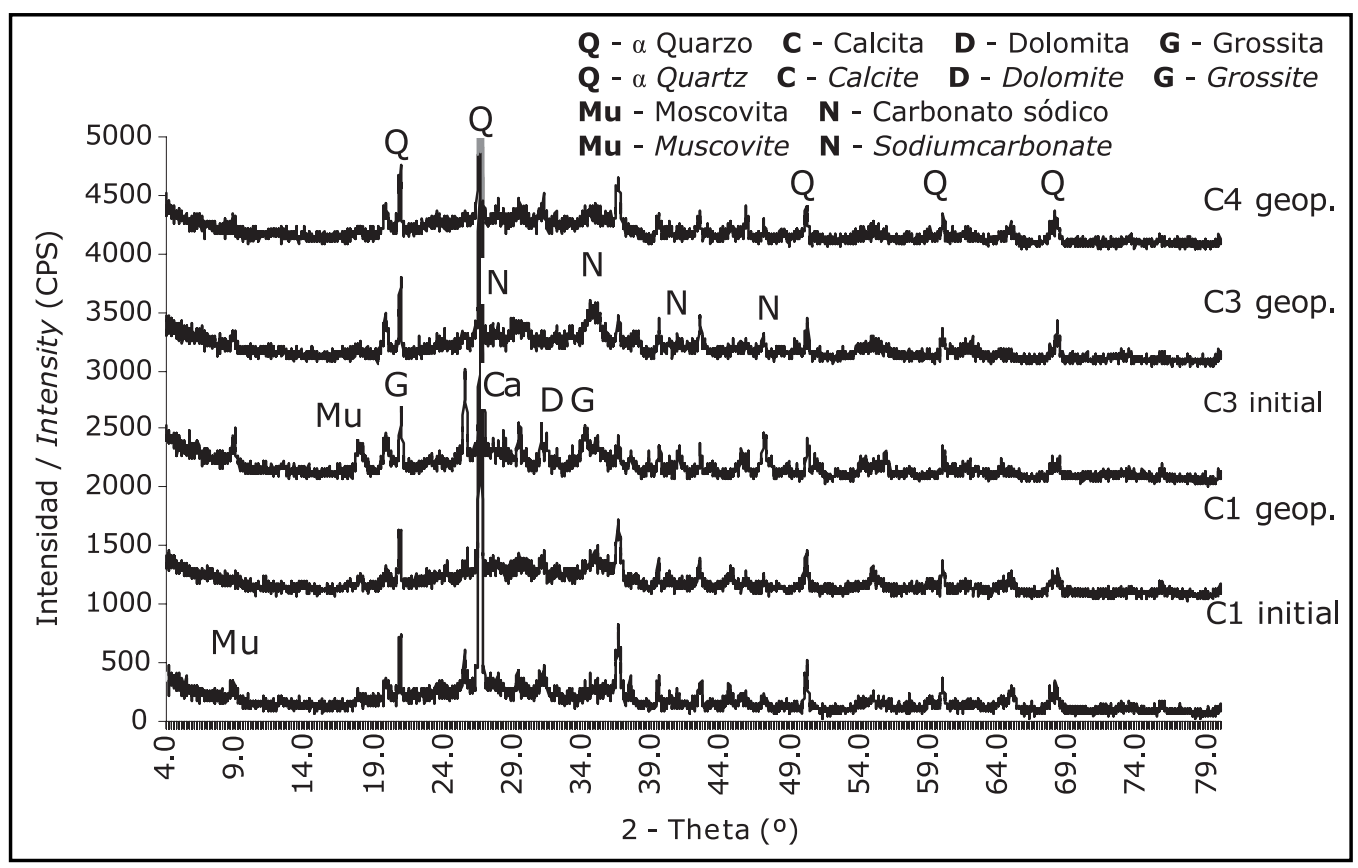

Figura 1. Espectros de DRX de las composiciones iniciales y después del proceso de geopolimerización. Figure 1. XXRD spectra for materials before (initial) and after (geop.) geopolymerization.

Con respecto a la caracterización mineralógica, el hecho de que los geopolímeros presentan un carácter débilmente cristalino o frecuentemente un carácter amorfo, limita el uso de la DRX para evaluar sus componentes, lo que permite, por otro lado, confirmar la reacción alcalina de activación (13). En particular, los picos de los minerales precursores de la arcilla deberían desaparecer o disminuirían con el avance del proceso. La figura 1 muestra los diagramas comparativos de DRX de los residuos de agregados ligeros antes (iniciales) y después del proceso de geopolimerización (geopolímero). Las fases principales que se han detectado en las muestras antes del la geo-
While XRD is able to provide only limited information on the constituents of geopolymers due to the amorphous or at best, weak crystalline - structure of these compounds, it can be used to confirm the existence of the alkali activation reaction (13). More specifically, the peaks attributed to the crystalline minerals in the clay should disappear or decline substantially as the reaction progresses. Figure 1 compares the XRD spectra for the LWA waste mixes before (initial) and after (geop.) geopolymerization. The main phases detected in the samples before geopolymerization were a-quartz $\left(\mathrm{SiO}_{2}\right)$, muscovite $\left(\mathrm{K}_{2} \mathrm{AL}_{4}\left(\mathrm{Si}_{6} \mathrm{Al}_{2} \mathrm{O}_{20}\right)(\mathrm{OH}){ }_{4}\right)$, grossite $\left(\mathrm{CaAl}_{4} \mathrm{O}_{7}\right)$, dolomite 
polimerización han sido: alfa-cuarzo, moscovita $\left(\mathrm{K}_{2} \mathrm{AL}_{4}\left(\mathrm{Si}_{6} \mathrm{Al}_{2} \mathrm{O}_{20}\right)(\mathrm{OH})_{-}\right)$, grosita $\left(\mathrm{CaAl}_{4} \mathrm{O}_{7}\right)$, dolomita $\left(\mathrm{MgCa}\left(\mathrm{CO}_{3}\right)_{2}\right)$ y calcita $\left(\mathrm{CaCO}_{3}\right)$. Todos estos compuestos están presentes o en las materias primas basadas en arcilla o en el proceso de producción de los áridos ligeros. Después de la geopolimerización, las fases principales son todavía alfa-cuarzo $\left(\mathrm{SiO}_{2}\right)$ y moscovita $\left(\mathrm{K}_{2} \mathrm{AL}_{4}\left(\mathrm{Si}_{6} \mathrm{Al}_{2} \mathrm{O}_{20}\right)\right.$ $\left.(\mathrm{OH})_{4}\right)$, pero aparece una cantidad pequeña de una nueva fase $\left(\mathrm{Na}_{2} \mathrm{CO}_{3}\right)$, relacionada con la presencia del sodio del activador. A pesar de la semejanza entre las muestras correspondientes respecto a lo que concierne a las fases cristalinas principales (p. ej., alfa- cuarzo), es posible confirmar la tendencia hacia la disminución de la intensidad de los picos relativos de las fases de silicato de aluminio en la arcilla (p. ej., alrededor de los ángulos $2 \theta=$ 9 y $25^{\circ}$ ). Además, todas las composiciones son razonablemente amorfas, como puede comprobarse por la banda de fondo entre los $20^{\circ}$ y $40^{\circ}$.

Las observaciones por SEM de la microestructura de los geopolímeros se muestran en la figura 2 para las composiciones $\mathrm{C} 1, \mathrm{C} 2, \mathrm{C} 3$ y C4. Estas microstructuras presentan imágenes bastantes típicas de los materiales geopoliméricos en muestras tomadas después de un mes de su fabricación. Es evidente en la matriz que los residuos de
$\left(\mathrm{MgCa}\left(\mathrm{CO}_{3}\right)_{2}\right)$ and calcite $\left(\mathrm{CaCO}_{3}\right)$. All these compounds were either present in the (clay-based) prime materials or introduced during LWA manufacture. The main phases after polymerization continued to be a-quartz $\left(\mathrm{SiO}_{2}\right)$ and muscovite $\left(\mathrm{K}_{2} \mathrm{AL}_{4}\left(\mathrm{Si}_{6} \mathrm{Al} \mathrm{O}_{2} \mathrm{O}_{20}\right)(\mathrm{OH})_{4}\right)$, but a new phase, namely $\mathrm{Na}_{2} \mathrm{CO}_{3}$, also appeared as a result of the presence of sodium in the activator. Despite the overall similarity of the diffractograms for the before and after sample pairs as regards the main crystalline phase (a-quartz), they confirmed the declining intensity of the aluminoussilicate clay-type phases (at around $2 \theta=90$ and 250) in the geopolymers. The mixes proved to be fairly amorphous, as shown by the typical halo between $20^{\circ}$ and $40^{\circ}$.

The SEM micrographs of geopolymers C1, C2, C3 and C4 are reproduced in Figure 2. The microstructures shown (scans for one-month samples) are typical of geopolymer materials. The images show that the powders with median diameters of under $27 \mathrm{~mm}$ interfaced well with the coarser particles (up to $3 \mathrm{~mm}$ in diameter). These
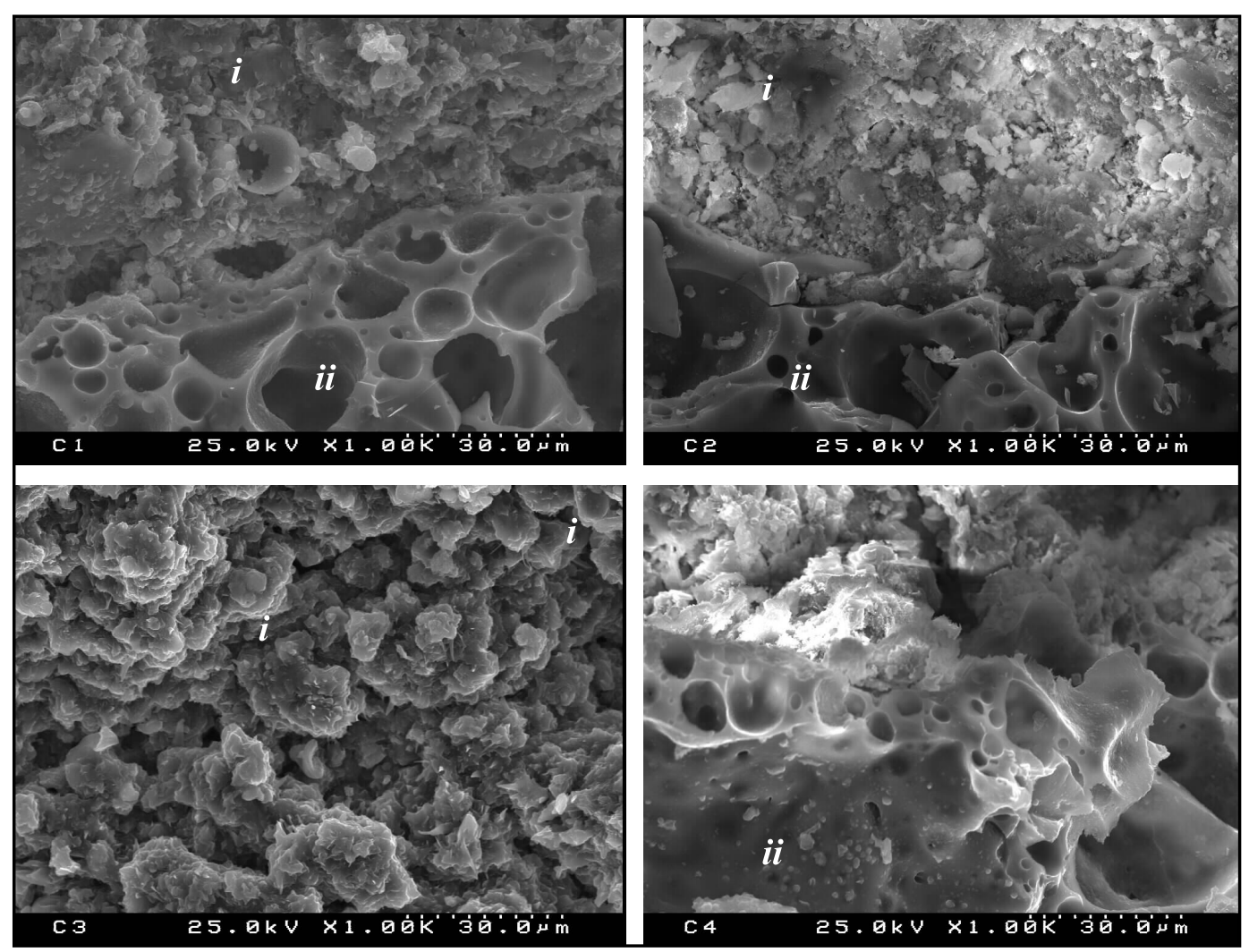

Figura 2. Microstructura de las composiciones de los geopolímeros ( $\mathrm{C} 1$ a 4 ): Polvos finos del residuo (i) y gránulos de áridos porosos (ii) dentro la matriz geopolimérica.

Figure 2. Geopolymer microstructure (C1 to C4): fine powder (i) and porous aggregate particles (ii) in the geopolymer matrix. 
polvos con un diámetro promedio menor de $27 \mu \mathrm{m}$ dan lugar a una buena interfase con los granos gruesos (por encima de $3 \mathrm{~mm}$ de diámetro). Sólo la composición C3 no presenta este tipo de partículas más gruesas en su formulación original, estando compuesta en su mayoría por los residuos de partículas más finas (polvos de aspiración y de tamizado). Esta composición es por tanto más densa que la anterior. Los áridos más gruesos presentes en las composiciones $\mathrm{C} 1, \mathrm{C} 2$ y $\mathrm{C} 4$ son bastantes porosos, lo que puede confirmarse en la figura 2 , en donde es también posible observar que hay una buena interfase de contacto entre las áreas de partículas más finas y más gruesas. Los datos adicionales obtenidos por EDX respecto a la relación $\mathrm{Si} / \mathrm{Al}$ en el gel deberían dar información importante sobre su composición pero no ha sido posible obtenerlos debido a las condiciones de tamaño y resolución de dichos sistemas complejos.

Con respecto a las propiedades mecánicas de estos materiales, las figuras 3 y 4 presentan los resultados de la resistencia a compresión y a flexión en diferentes composiciones con diversos tiempos de curado. coarser particles were lacking in paste C3, whose source materials contained finer particles only (filter and sieve powder). And this was, in fact, the densest of the four pastes. As Figure 2 shows, the coarse aggregates in pastes C1, C2 and C4 were quite porous and it is also possible to observe a good interface between finer and coarser particles. While EDX gives rise for information on the $\mathrm{Si} / \mathrm{Al}$ ratio, the accuracy of this technique is compromised when applied to such complex systems.

The results of the compressive and bending strength tests conducted on these materials at different ages are shown in Figures 3 and 4.

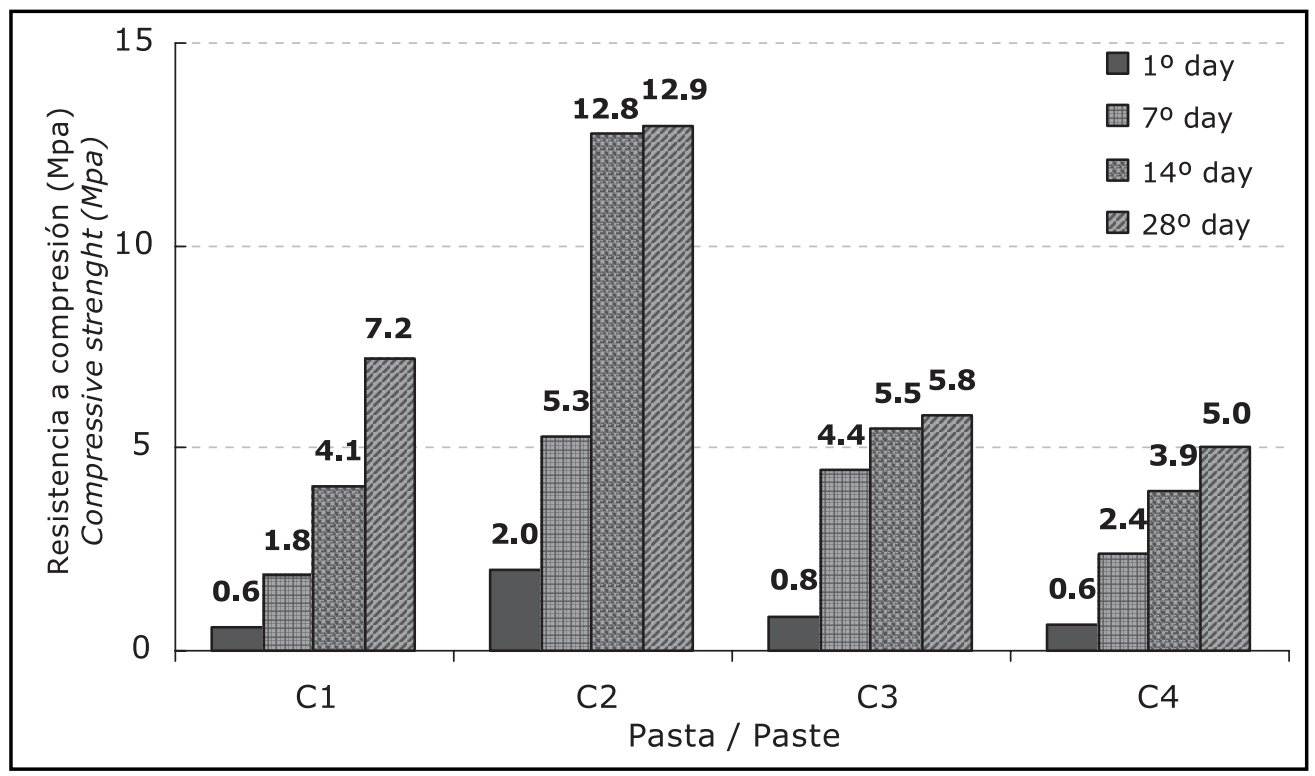

Figura 3. Valores de resistencia a compresión.

Figure 3. Compressive strength values.

Como era de esperar, la resistencia mecánica del cemento basado en la composición C2 es más elevada que los cementos de residuos basados en los geopolímeros $\mathrm{C} 1$, C3, y C4. Independientemente del hecho de que el sistema agua- cemento da lugar a una resistencia mayor, se debería considerar la introducción de áridos porosos en las otras composiciones. No obstante, los valores logrados tanto para la resistencia a flexión como a compresión pueden ser interesantes para algunas aplicaciones espe-
The mechanical strength values for the cement-based paste (C2) were, as expected, higher than for the wastebased geopolymers (C1, C3 and C4). While the cementwater system developed higher strength, the effect of including porous aggregates in the other pastes should not be overlooked. In any case, the compressive and bending strength values attained by the geopolymers may suffice for certain applications, and could possibly be 
cíficas y podrían ser mejorados controlando las condiciones de curado y/ o las composiciones $(13,14)$. enhanced by controlling curing conditions and/or composition $(13,14)$.

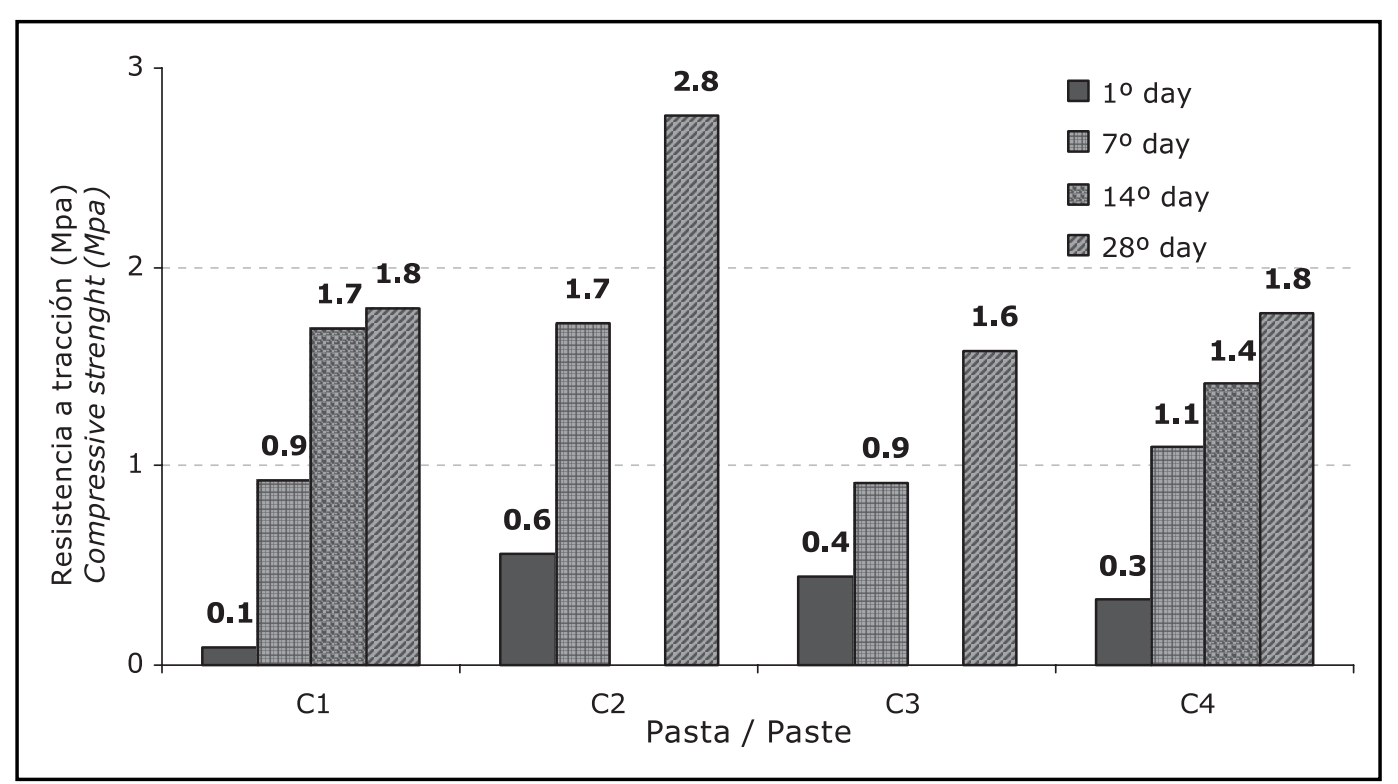

Figura 4. Valores de resistencia a flexión.

Figure 4. Bending strength values.

La concentración del activador alcalino usado (5M y 10M) no da lugar a ninguna diferencia específica en la resistencia mecánica como puede verse comparando las composiciones $\mathrm{C} 1$ y $\mathrm{C} 4$. Aunque, parece ser hay una clara ventaja cuando es menor la concentración del activador (C4), lo que está relacionado con una trabajabilidad durante mayor tiempo, que es muy importante para el mezclado homogéneo y el moldeado de las muestras.

Este geopolímero C4, que es el que contiene los áridos más gruesos y porosos, parece el más prometedor para futuras aplicaciones que demanden un cierto carácter aislante. Desde este punto de vista, se ha realizado una caracterización preliminar y se ha obtenido, para este producto, una densidad de $1,23 \mathrm{~g} / \mathrm{cm}^{3}$ dando el coeficiente de conductividad térmica un valor de $0,41 \mathrm{Wm}^{-1} \mathrm{~K}$ 1 que se considera interesante. Aumentando la cantidad de áridos porosos se reducirá por supuesto este valor, pero disminuirá también el valor de la resistencia mecánica, lo que significa que un compromiso debe buscarse en función de la aplicación deseada.

Hay formación de sales que tienen lugar en algunas de las composiciones de geopolímeros durante el tiempo de curado, especialmente en las C3 y C4. Las sales fueron raspadas de la superficie de estas muestras y se analizaron por DRX (figura 5). Los resultados para ambas muestras fueron coincidentes y la fase detectada fue un carbonato de sodio $\left(\mathrm{Na}_{2} \mathrm{CO}_{3} \cdot \mathrm{H}_{2} \mathrm{O}\right)$. Investigaciones posteriores
A comparison of the findings for pastes $C 1$ and C4 showed that the concentration of the alkaline activator (10or 5-M) had no significant effect on mechanical strength. Nonetheless, one clear advantage of using a lower concentration (C4) was identified: the paste retained its workability longer, a very important consideration as far as thorough mixing and effective paste moulding are concerned.

Inasmuch as geopolymer C4, which contained coarser, porous aggregate, appeared to be promising for use as insulation, preliminary tests were conducted to determine its average density $\left(1.23 \mathrm{~g} / \mathrm{cm}^{3}\right)$ and thermal conductivity $\left(0.41 \mathrm{Wm}^{-1} \mathrm{~K}^{-1}\right)$. Raising the proportion of porous aggregates would reduce those values, but would logically also prompt a decline in mechanical strength. A compromise would have to be sought, depending on the application.

Salts formed in some of the geopolymers, primarily C3 and C4, during curing. When they were removed from the sample surface and analyzed with XRD (Figure 5), the phase identified in both $\mathrm{C} 3$ and $\mathrm{C} 4$ was a sodium carbonate $\left(\mathrm{Na}_{2} \mathrm{CO}_{3} \cdot \mathrm{H}_{2} \mathrm{O}\right)$. Further studies will be conducted to eliminate this problem, whose solution may consist in 
llevarán a eliminar este problema que podría ser resuelto por la selección apropiada de las condiciones de curado y también cambiando la concentración de sodio con el fin de prevenir la formación de carbonato (15). refining the curing conditions or changing the concentration of the activator (15).

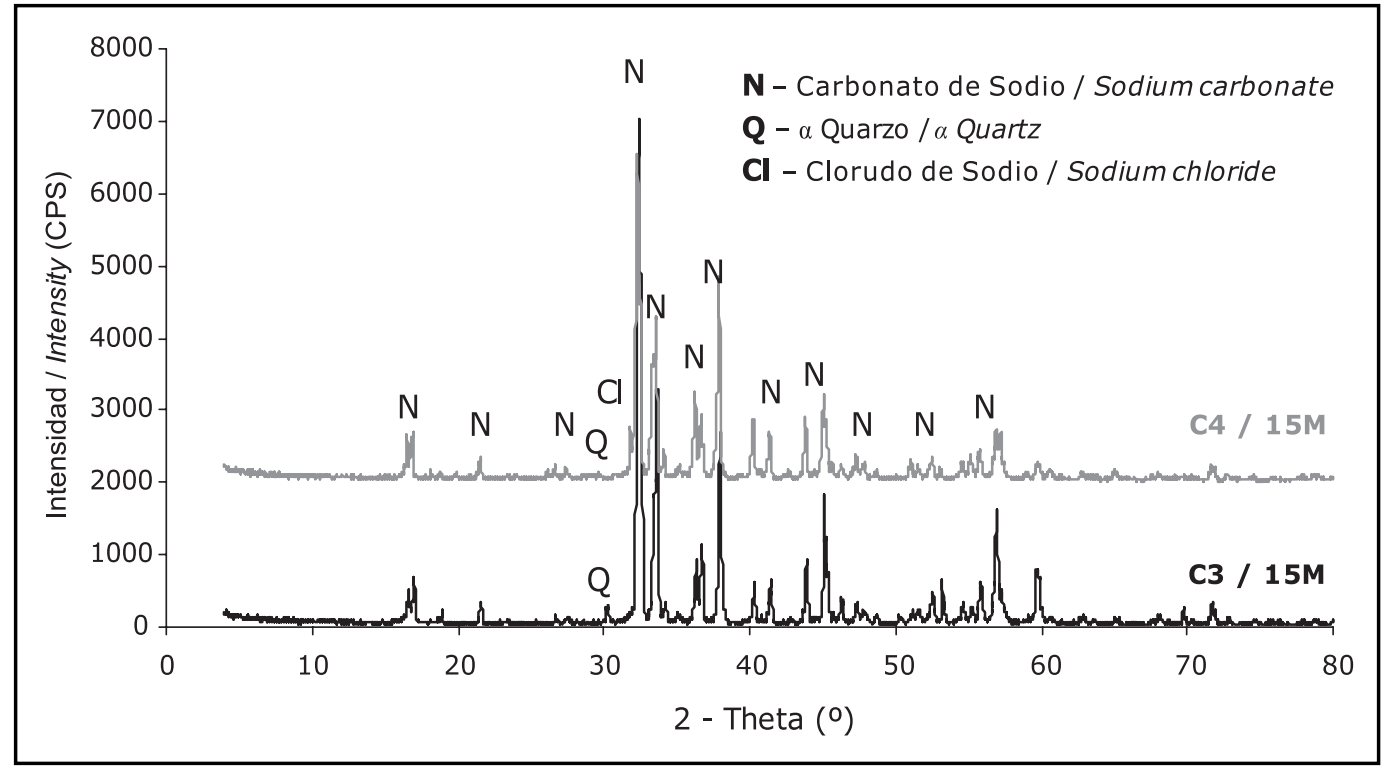

Figura 5. Análisis por DRX de las sales raspadas en las superficies de los geopolímeros C3 y C4. La concentración del activador de hidróxido de sodio ha sido del 15M en ambos casos.

Figure 5. XRD analysis of salts removed from geopolymers C3 and C4. The sodium hydroxide activator concentration was $15 \mathrm{M}$ in both cases.

\section{CONCLUSIONES}

Los resultados muestran que los residuos de este estudio son adecuados para producir productos de geopolímeros para aplicaciones en la construcción. Los residuos que proceden de la fabricación de áridos ligeros basados en arcillas presentan unos niveles adecuados de $\mathrm{SiO}_{2}$ y $\mathrm{Al}_{2} \mathrm{O}_{3}$. Además de permitir la producción de un nuevo material, esta solución también contribuye para valorizar y eliminar estos residuos, que de otra manera constituirían un problema ambiental.

Ha sido también posible determinar que disminuyendo la concentración del activador alcalino la trabajabilidad o "tiempo abierto" para el curado puede ser ampliada, facilitando el proceso de mezclado y moldeado, sin degradar las propiedades mecánicas. Cambiando el tipo de residuo procedente de la fabricación de áridos ligeros y usando una cantidad más alta de fracción porosa $0 / 3$ ha sido posible conseguir una composición de un geopolímero (C4) con características aislantes interesantes que merece un estudio adicional de optimización. Aunque pueden producirse algunas sales, se cree que la modificación de las condiciones de curado eliminarían este problema.

\section{CONCLUSIONS}

The results showed that the waste from lightweight claybased aggregate manufacture studied here are adequate for producing geopolymers that can be used in building materials. These industrial by-products proved to have suitable proportions of $\mathrm{SiO}_{2}$ and $\mathrm{Al}_{2} \mathrm{O}_{3}$. In addition to generating a new material, this solution provides for the re-use and elimination of such waste, which would otherwise constitute an environmental problem.

Decreasing the concentration of the alkaline activator was found to widen the workability window of the pastes, facilitating mixing and moulding with no detriment to mechanical strength. Changing the type of LWA waste by including larger proportions of the porous 0/3- $\mathrm{mm}$ fraction yielded a geopolymer with promising insulating characteristics that merits further study and optimization. While some salt formation was observed, this problem may very likely be solved by modifying curing conditions. 


\section{AGRADECIMIENTOS}

Los autores desean de reconocer el apoyo dado a este trabajo por el programa PRAI administrado por la Comisión Portuguesa de Coordinación del Desarrollo de la Región Centro.

\section{ACKNOWLEDGEMENTS}

This study was supported by the Portuguese Centre Region Coordination and Development Commission's PRAI Programme.

\section{BIBLIOGRAFÍA / BIBLIOGRAPHY}

(1) Pinto S., Almeida M., Correia A.M.S., Labrincha J.A., Ferreira V.M., Rosenbom, K.: "Use of recycled materials in buildings and structures". Proceedings of International RILEM Conference on the use of recycled materials in buildings and structures, Vol. 2 (2004), pp. 771-777.

(2) Dondi M., Marsigli M., Fabbri B.: "Recycling of urban and industrial wastes in brick production: a review". Tile \& Brick Int., Vol. 13, n04 (1997), pp. 302-315.

(3) Tay J.H., Show K.Y., Hong S.Y.: "Reuse of industrial sludge as construction aggregates". Water Science Technology, Vol. 44, n¹0 (2001), pp. 269-272.

(4) Hua X., Van Deventer J.S.J.: "The geopolymerisation of alumino-silicate minerals". Int. J. Min. Proc., Vol. 59 (2000), pp. $247-266$. http://dx.doi.org/10.1016/S0301-7516(99)00074-5

(5) Hua X., Van Deventer J.S.J.: "Geopolymerisation of multiple minerals". Minerals Engineering, Vol. 15 (2002), pp. 1131-1139. http://dx.doi.org/10.1016/S0892-6875(02)00050-X

(6) Davidovits J.: "Geopolymer chemistry and properties". Proceedings of First European Conference on Soft Mineralurgy, (1988), pp. 25-48.

(7) Palomo A., Macías A., Blanco M.T., Puertas F.: "Physical chemical and mechanical characterization of geopolymers". Proceedings of 9th International Congress on the Chemistry of Cement, (1992), pp. 505-511.

(8) Comrie D.C., Patersen J.H., Ritcey D.J.: "Geopolymer applications in the mining industry". Preprint 20th Canadian Mineral Processors Annual Operators Conference (1988).

(9) Davidovits J.: US Patent Application 104.190 (1987).

(10) Davidovits J., Comrie D.: "Long term durability of hazardous toxic and nuclear waste disposals". Proceedings of First European Conference on Soft Mineralurgy (1988), pp. 125-134.

(11) Davidovits J, Comrie D.: "Geopolymeric reactions in archaeological cements and in modern blended cements". Proceedings of First European Conference on Soft Mineralurgy (1988), pp. 93-106.

(12) Palomo A., Alonso S.: "Calorimetric study of alkaline activation of calcium hydroxide-metakaolin solid mixtures". Cem. Concr. Res., Vol. 31 (2001), pp. 25-30.

(13) Palomo A., Alonso S., Fernández J.A., Sobrados I., Sans J.: "Alkaline activation of fly ashes. NMR study of the reaction products". J. Amer. Ceram. Soc., Vol. 87, n'6 (2004), pp. 1141-1145.

(14) Fernández J. A., Palomo A.: "Characterisation of fly ashes. Potential reactivity as alkaline cements". Fuel, Vol. 82 (2003), pp. $2259-2265$.

(15) Palomo A., Fernández J.A.: "Alkaline activation of fly ashes. Manufacture of concretes not containing Portland cement". Proceedings of International RILEM Conference on the use of recycled materials in buildings and structures, Vol. 2 (2004), pp. 863-870. 